the fibromiyalgia and rheumatoid arthritis, healthy blood donors $(\mathrm{p}>0.05)$.

Conclusion In conclusion, our study suggest that there may exist an association between fibromiyalgia and HBs-Ag. In addition, our data do not support the hypothesis that HCV infection may play a role in the pathogenesis of fibromyalgia.

\section{SAT0136 CHEST EXPANSION AND SPIROMETRIC MEASURES IN PATIENTS WITH PRIMARY FIBROMYALGIA}

${ }^{1} S$ Ozgocmen, ${ }^{2} \mathrm{O}$ Bolgen Cimen, ${ }^{1} \mathrm{O}$ Ardicoglu. ${ }^{1}$ Physical Medicine and Rehabilitation, Firat University, Faculty of Medicine, Elazig; ${ }^{2}$ Physical Medicine and Rehabilitation, Mersin University, Faculty of Medicine, Mersin, Turkey

10.1136/annrheumdis-2001.595

Background Despite normal spirometric values patients with fibromyalgia had lower values of maximum inspiratory (MIP) and expiratory pressures (MEP) than matched healthy controls. Values of MIP and MEP reflect respiratory muscle strength, therefore low values have been explained by respiratory muscle weakness or dysfunction.

Objectives This study proposed to assess the relationship of chest expansion with maximal inspiratory (MIP) and expiratory pressures (MEP) in primary fibromyalgia (FM) syndrome.

Methods Thirty female patients, aged 22-52, who met the 1990 ACR [1] criteria for the classification of FM were examined and compared with 29 healthy, age and BMI matched female subjects (aged 24-50). Pulmonary function tests were assessed by a computerised spirometer. The maximum respiratory pressures were carried out by using a digital mouth-pressuremeter. Chest expansion measurements, spirometric values, MIP and MEP values of patients with primary FM have been compared with controls.

Results Patients with FM had lower chest expansion, MEP and MIP values with respect to the controls. Chest expansion correlated significantly with MIP and MEP values. There was no significant difference among groups in spirometric values.

Conclusion Our results indicate that patients with FM have impaired respiratory muscle strength and measurement of chest expansion may be an appropriate and practical method for evaluating respiratory muscle strength in patients with FM.

\section{SAT0137 LONG-TERM OBSERVATION OF FIBROMYALGIA PATIENTS}

L Szczepanski, C Stepniak, B Targonska-Stepniak. Department of Rheumatology, University Medical School in Lublin, Poland, Lublin, Poland

\subsection{6/annrheumdis-2001.596}

\section{Background}

Objectives The objective of the study was to evaluate the natural course of fibromyalgia syndrome (FS) and to reveal if FS was not only the mask of another somatic disease.

Methods A questionnaire concerning the present health state was sent to 177 patients with FS diagnosed between 1991 and 1997. One hundred fifteen answers were received. Thirty three patients $(28,7 \%)$ had no pain. In $19(16,5 \%)$ cases the same intensity of pain and other subjective symptoms were noted. Majority of patients- $63(54,8 \%)$ assessed their complaints as more intensive. Clinical and basic laboratory examinations were performed in 86 FS women, aged 39-70. They were asked about their present pain status, other complaints, mental status and their understanding of the matter of FS. Moreover, in all cases the FIQ answers were obtained. In some cases additional tests were performed (X-rays, Schrimer?s test, ANA, RF).

Results At examination only 5 patients $(5,8 \%)$ had no pain in musculoskeletal system and felt very well; in the remaining 81 patients the features of active FS were noted. 43 patients $(50 \%)$ described the pain as ?awful" and ?terrible?, in 32 cases $(37,2 \%)$ the pain was great or even insufferable. Less than 11 tender points (TPs) in 11 patients $(12,8 \%)$, however in these patients other manifestations of FS were present (sleep disturbances, fatigue, ancillary symptoms). In the remaining 70 patients all ACR criteria for diagnosis of FS were still present.

Four patients $(4,7 \%)$ fulfilled criteria for diagnosis of Sjögren's Syndrome (SS); ocular and oral dryness appeared later than widespread pain. There were no features of other connective tissue diseases in the remaining 82 patients. At examination other musculosceletal disorders were observed in some cases: gonarthrosis (4 patients- 4,7\%), coxarthrosis (6 patients- 7\%), carpal tunnel syndrome (21 patients- $24,4 \%)$, enthesopathy (15 patients- $17,4 \%)$. The majority of FS patients (61- 70,9\%) still felt suspicion of severe somatic disease and were anxious about the future; 44 patients $(51,2 \%)$ believed, that careful hospital examination is necessary.

Conclusion FS remains active in most patients several years after the first diagnosis. The majority of patients feel poor or even worse, the remission of FS is uncommonly achieved and the results of treatment are still unsatisfactory. No serious chronic diseases were observed in those patients. The results confirm the high usefulness of ACR 1990 FS criteria in everyday clinical practice. In a few cases FS can be associated with subclinical course of SS.

\section{SAT0138 SELF-REPORTED PAIN AND SLEEP QUALITY IN PATIENTS WITH FIBROMYALGIA}

${ }^{1} \mathrm{AE}$ Corbin, ${ }^{1} \mathrm{M}$ Jaffe, ${ }^{2} \mathrm{~J}$ Moore, ${ }^{2} \mathrm{JP}$ Young, ${ }^{2} \mathrm{LK}$ LaMoreaux, ${ }^{3} \mathrm{DC}$ Turk, ${ }^{1} \mathrm{U}$ Sharma, ${ }^{4} \mathrm{M}$ Versavel. ' CNS Clinical Development; ${ }^{2}$ Clinical Statistics, Pfizer Global R \& D, Ann Arbor, MI, USA; ${ }^{3}$ Department of Anesthesiology, University of Washington, Seattle WA, USA; ${ }^{4}$ CNS Clinical Development, Pfizer Global $R \& D$, Fresnes, France

\subsection{6/annrheumdis-2001.597}

Background Chronic widespread pain and sleep disturbance are among the most common symptoms reported by patients with fibromyalgia (FM).

Objectives This study assessed the level of pain and sleep disturbance in FM patients using a daily pain and sleep quality diary, the Short-Form McGill Pain Questionnaire (SF-MPQ), and the Manual Tender Point Survey (MTPS).

Methods Patients recorded their levels of pain severity and sleep quality for approximately 7 days using an 11-point numerical rating scale (ranging from $0=$ no pain/best possible sleep to 10 = worst possible pain/sleep, respectively). The SF-MPQ measured pain quality and severity (range $0-3$ ) with 11 sensory and 4 affective descriptors, and pain severity using a $100 \mathrm{~mm}$ visual analogue scale (VAS). The MTPS assessed the presence of pain at each of 18 tender points corresponding to those specified by the American College of Rheumatology (ACR) 1990, and 3 control points. Patients rated the severity of pain upon palpation of each of the tender and control points on a $0-10$ point scale (Tender Point Pain Intensity Score). These measures were administered to 529 patients diagnosed with FM using the ACR criteria during the baseline period of a clinical drug trial. The mean age was 48.6 years, $91.5 \%$ were female, and the mean duration of FM was 107.7 months. 
Results Patients with FM reported a mean pain score of 7.0 $(+1.3)$ and mean sleep quality score of $6.5(+1.7)$ on the $0-10$ point scale. Over $95 \%$ of patients selected the descriptors aching, tender and tiring-exhausting. The mean pain score as measured by the VAS of the SF-MPQ was $74.8(+14.2)$ on the $0-100$ point scale. The mean number of tender points was $17.1(+1.5)$ of 18 possible; the mean Tender Point Pain Intensity Score (010 point scale) was $6.1(+1.8)$, while control point mean pain severity was $2.0(+1.9)$.

Conclusion Patients with FM reported a moderate to severe level of pain and sleep disturbance. Pain severity was consistently reported across the three measures of pain, the daily pain diary, the VAS, and the Tender Point Pain Intensity Score. Nearly all patients consistently described their pain as tender, aching and tiring-exhausting. Additional studies need to be completed to characterise further the pain and sleep disturbance prevalent in patients with FM, and to assess and define other symptoms involved in the FM syndrome (see companion abstract by Sesti, et al.).

\section{SAT0139 A CONTROLLED LONG-TERM TREATMENT OF FIBROMYALGIA PATIENTS WITH SERTRALINE}

${ }^{1} \mathrm{P}$ Sarzi-Puttini, ${ }^{2} \mathrm{R}$ Pettorossi, ${ }^{2} \mathrm{~A}$ Baffigi, ${ }^{1} \mathrm{M}$ Cazzola, ${ }^{1} \mathrm{~T}$ Fiorini. ${ }^{2}$ Rheumatology, L. Sacco; ${ }^{2}$ Unit and Laboratory of Clinical Psychology, Catholic University of Sacred Heart, Milano, Italy

\subsection{6/annrheumdis-2001.598}

Background The pathophysiology of Fibromyalgia syndrome (FM) is mostly uncertain and the conventional medication therapy results from empirical research. FM is a common disorder that is estimated to affect $2-4 \%$ of the population. Serotonin reuptake inhibitors are now used in FM due to their interference in the serotonin metabolism.

Objectives To evaluate the relative efficacy and tolerability of Sertraline in the long-term treatment of fibromyalgia patients.

Methods Fifty patients who fulfilled the American College of Rheumatology criteria for the classification of fibromyalgia were entered in a 6-month prospective, controlled trial; all patients were treated with Sertraline (50-100 mg/day). Symptomatic treatment with nonsteroidal anti-inflammatory drugs (NSAIDs) and/or analgesic was allowed at optimal doses that were established on the basis of each patient?s needs. Five control examinations were planned (at baseline, after 15 days at month 1,2,3, and 6). Primary efficacy measures were (1) patient self assessment of pain on a $10 \mathrm{~cm}$ visual analogue scale (VAS), (2) tender point index (TPI) which is the sum of individual tenderness severity at each of the 18 standard ACR tender points and (3) Fibromyalgia Impact Questionnaire (FIQ). Other six secondary clinical efficacy measures were selected: physician and patients' global assessment of the disease, sleep quality assessment using a 100-mm visual analogue scale, Health Assessment Questionnaire (HAQ) the Hamilton rating scale for depression (HRSD) and the Hamilton rating scale for anxiety (HRSA). Tolerability assessment was based on the occurrence of any adverse events.

Results All primary efficacy measures were significantly improved after 1 month of treatment $(p<0.05)$ and maintained a significant improvement throughout the study. No further improvement was however observed after 3 months of treatment. Most of the secondary efficacy measures improved significantly; in particular HRDS and HRSA $(\mathrm{p}<0.01)$ improved after 1 month of treatment. After 6 months of treatment, 23 patients $(45 \%)$ were still on treatment with Sertraline. Most of the withdrawals were due either to inefficacy (12 patients), sideeffects (7 patients) or lost to follow-up (8 patients).

Conclusion Our data confirm the long-term efficacy of sertraline in a discrete percentage of patients with fibromyalgia.

\section{SAT0140 HYPERSOMNOLENCE IN FIBROMYALGIA SYNDROME}

M Rizzi, P Sarzi-Puttini, A Andreoli, B Panni, M Pecis, FG Vassallo, M Carrabba, M Sergi. Servizio Di Fisiopatologia Respiratoria, L. Sacco, Milano, Italy

10.1136/annrheumdis-2001.599

\section{Background}

Objectives To evaluate hypersomnolence in patients affected by fibromyalgia syndrome.

Methods Thirty consecutive patients affected by fibromyalgia syndrome (FMS) (28 F) underwent a general sleep questionnaire, the Epsworth sleepiness scale (ESS), formal polysomnography, and lung function tests.

Results Patients complaining of daytime hypersomnolence had a higher number of tender points $(15 \pm 2$ vs $12 \pm 1$, p $<0.01)$, a greater score of subjective pain $(72 \pm 15$ vs $52 \pm 13, \mathrm{p}=0.05)$ and more fatigue $(\mathrm{p}=0.04)$, about twice as many arousals per hour and a lower sleep efficiency than patients who did not report this symptom. TLco was more impaired and the occurrence of $\mathrm{PB}$ higher.

FMS patients who referred daytime somnolence slept significantly less efficiently than the FMS with no day time somnolence $(\mathrm{p}<0.05)$, had a lower proportion of stage 3 sleep $(5 \pm$ $2 \%$ vs $12 \pm 3 \% ; \mathrm{p}<0.001)$, stage $4(1 \pm 0.5 \%$ vs $4 \pm 1 \%$; $<0.001$ ), and twice as many arousals per hour of sleep ( $\mathrm{p}<$ $0.01)$. The respiratory pattern of FMS patients with hypersomnolence showed a higher occurrence of periodic breathing ( $p=$ 0.02). The short length of apneas and hypopneas did not affect the apnea/hypopnea index $(5.1 \pm 3$ vs $7 \pm 4 ; \mathrm{p}=\mathrm{ns})$, but FMS patients with daytime hypersomnolence had a greater number of desaturations per hour of sleep $(11 \pm 6$ vs $6 \pm 5$; p < 0.05). Pulmonary volumes did not differ between the two groups.

Conclusion The occurrence of daytime hypersomnolence in FMS patients, is linked to a greater severity of fibromyalgia symptoms and to a more severe polisomnographic alterations.

\section{SAT0141 THE EFFECTS OF MIRTAZAPINE IN THE TREATMENT OF FIBROMYALGIA SYNDROME}

W Samborski, M Lezañska-Szpera, JK Rybakowski. Rheumatology, K. Marcinkowski University Medical School, Poznan, Poland

\subsection{6/annrheumdis-2001.600}

Background Some previous study has documented the efficacy of 5-HT3 receptors antagonists in the treatment of fibromyalgia. Objectives The mirtazapine (Remeron), a new generation antidepressive agent, characterised by blocking of 5-HT2 and 5-HT3 receptors and stimulation of 5HT-1 and noradrenergic receptors was used in the treatment of patients with Fibromyalgia syndrome (FS).

Methods Twenty patients (18 female, 2 male), age range 20-64 years, mean 43,58, fulfilling the 1990 ACR criteria for fibromyalgia, entered 6 weeks open study and were given $30 \mathrm{mg}$ mirtazapine per day. Before the administration of the first dose, during the study and at the final visite, the following parameters were measured using the visual analogue scale 0-10 (VAS): pain, 